\title{
Ultrasound-evoked immediate early gene expression in the brainstem of the Chinese torrent frog, Odorrana tormota
}

\author{
Victoria S. Arch · Sabrina S. Burmeister • \\ Albert S. Feng $\cdot$ Jun-Xian Shen $\cdot$ Peter M. Narins
}

Received: 13 May 2010/Revised: 16 January 2011/ Accepted: 17 January 2011/Published online: 5 February 2011

(C) The Author(s) 2011. This article is published with open access at Springerlink.com

\begin{abstract}
The concave-eared torrent frog, Odorrana tormota, has evolved the extraordinary ability to communicate ultrasonically (i.e., using frequencies $>20 \mathrm{kHz}$ ), and electrophysiological experiments have demonstrated that neurons in the frog's midbrain (torus semicircularis) respond to frequencies up to $34 \mathrm{kHz}$. However, at this time, it is unclear which region(s) of the torus and what other brainstem nuclei are involved in the detection of ultrasound. To gain insight into the anatomical substrate of ultrasound detection, we mapped expression of the activity-dependent gene, egr-1, in the brain in response to a full-spectrum
\end{abstract}

\section{S. Arch ( $₫)$}

Department of Biology, Stanford University,

Stanford, CA 94305, USA

e-mail: varch@stanford.edu

\section{S. S. Burmeister}

Department of Biology, University of North Carolina,

Chapel Hill, Chapel Hill, NC 27599, USA

\section{A. S. Feng}

Department of Molecular and Integrative Physiology,

University of Illinois, Urbana-Champaign,

Urbana, IL 61801, USA

\section{J.-X. Shen}

State Key Laboratory of Brain and Cognitive Science, Institute of Biophysics, Chinese Academy of Sciences, Beijing 100101, China

\section{P. M. Narins}

Department of Ecology and Evolutionary Biology, University of California, Los Angeles, Los Angeles, CA 90095, USA

\section{P. M. Narins}

Department of Integrative Biology and Physiology, University of California, Los Angeles, Los Angeles, CA 90095, USA mating call, a filtered, ultrasound-only call, and no sound. We found that the ultrasound-only call elicited egr-1 expression in the superior olivary and principal nucleus of the torus semicircularis. In sampled areas of the principal nucleus, the ultrasound-only call tended to evoke higher egr-1 expression than the full-spectrum call and, in the center of the nucleus, induced significantly higher egr-1 levels than the no-sound control. In the superior olivary nucleus, the full-spectrum and ultrasound-only calls evoked similar levels of expression that were significantly greater than the control, and egr-1 induction in the laminar nucleus showed no evidence of acoustic modulation. These data suggest that the sampled areas of the principal nucleus are among the regions sensitive to ultrasound in this species.

Keywords Ultrasonic communication - Anuran amphibian · Sensory physiology $\cdot$ Playback · China

\author{
Abbreviations \\ BP Basilar papilla \\ TS Torus semicircularis \\ Ptor Principal nucleus of the torus semicircularis \\ cPtor Central sampling region of the principal nucleus \\ dPtor Dorsal sampling region of the principal nucleus \\ Ltor Laminar nucleus of the torus semicircularis \\ SON Superior olivary nucleus
}

\section{Introduction}

All terrestrial vertebrate classes have evolved neural systems for detecting and processing airborne sound. Comparative studies of auditory system structure and function, 
within and across taxa, have provided key insights into neural diversity resulting from distinct evolutionary pressures. Such studies also offer the possibility of identifying mechanisms fundamental to all terrestrial hearing. Anuran amphibians (frogs and toads) are a good model for comparative research on sonic signal detection and processing because the auditory systems of anurans and mammals, including humans, share many functional similarities. However, anurans typically have small, highly stereotyped vocal repertoires, and the salient features of their calls are often well characterized. Thus, anurans provide a tractable model in which it is quite feasible to relate call parameters and concomitant nervous system processing to the animals' natural behavior.

Although anurans and mammals employ comparable mechanisms for reception and transduction of sound, differences between the peripheral apparatus of amphibians and mammals have been thought to place functional limitations on anuran hearing. Because anurans lack derived characteristics of the mammalian middle ear and cochlea that are implicated in high-frequency sensitivity (Fettiplace and Fuchs 1999), they have been considered to be restricted to hearing below approximately $5-8 \mathrm{kHz}$; in contrast, the majority of mammals hears well into the ultrasonic range (i.e., $>20 \mathrm{kHz}$ ). However, it was recently discovered that male concave-eared torrent frogs (Odorrana tormota) produce calls with substantial harmonic energy in the ultrasonic frequency range (Narins et al. 2004). Electrophysiological recordings from the torus semicircularis (TS; amphibian homologue of the mammalian inferior colliculus) demonstrated that neurons therein respond to audible as well as ultrasonic frequencies up to $34 \mathrm{kHz}$ (Feng et al. 2006). Behavioral studies verify that the high-frequency signal components can be used for intermale communication: male frogs call antiphonally in response to playback of conspecific calls high-pass filtered to contain only ultrasounds (Feng et al. 2006). The concave-eared torrent frog has unusual peripheral adaptations that may play a role in facilitating the transmission of high frequencies through the middle ear. Most strikingly, the frogs' tympanic membranes are located in chambers in the side of the head (Feng et al. 2006). Recessing the eardrums into the skull, and thus closer to the inner ear, may allow the frogs to have shorter, lighter middle ear ossicles that can transmit high frequencies more effectively. In addition, the species' tympanic membranes are ultra-thin, making them less massive, and thus more capable of high-frequency vibration (Feng et al. 2006). The mechanisms underlying the extraordinary high-frequency sensitivity of the frogs' inner ear and central auditory system, however, are uncharacterized.

Ultrasonic hearing requires that ultrasounds not only are effectively transmitted to the inner ear, but also that hair cells in the inner ear, and neurons in the brain, process signals in the extended frequency range. Preliminary investigations of the inner-ear auditory morphology of male concave-eared torrent frogs suggest that the frogs' high-frequency sensitivity has evolved through a mosaic of somewhat subtle modifications to the typical anuran auditory endorgan structure, rather than through a dramatic reorganization of the inner-ear auditory apparatus (Arch 2010). For example, in a direct comparison between the inner ears of Rana pipiens (a non-ultrasonically sensitive frog), and O. tormota, the basilar papilla (BP) hair cells of the latter exhibit shorter soma lengths, shorter hair bundle heights, and are fewer in number than in the BP of the former (Arch 2010). The gross structure of the organ, however, remains quite similar in both species. It is unknown if there are parallel differences in their central auditory systems. Unlike the peripheral auditory system, physiological and anatomical data indicate that the brainstem auditory pathways of anurans and mammals have a common origin (Wilczynski 1988). Thus, it is of comparative interest to explore the means by which highfrequency encoding has been incorporated into the $O$. tormota brain. This exploration could shed light on the possible evolutionary pathways through which nearly all mammals attained high-frequency sensitivity.

To gain insight into the structures responsible for ultrasound sensitivity in the frog brain, we examined neural activity patterns in the auditory brainstem of $O$. tormota in response to a full-spectrum conspecific call, a filtered, ultrasound-only call, and no sound. We assessed neural activity by measuring the expression of the immediate early gene, egr-1. Egr-1 mRNA is expressed in many neurons in response to depolarization (Clayton 2000) and therefore can serve as an activity marker (Hoke et al. 2004; Jarvis 2004). We measured egr-1 expression in the superior olivary nucleus (SON) in the hindbrain, which is a major source of afferents for the midbrain TS, and in the principal (Ptor) and laminar (Ltor) nuclei of the TS. We found that ultrasound-only calls elicited robust expression of $e g r-1$ in the SON and Ptor. In the Ptor, our metric for egr-1 expression tended to show a greater value in response to the ultrasound-only call than the full-spectrum call, suggesting that this nucleus may be important for the detection of ultrasound in this species.

\section{Methods}

Stimulus preparation

We selected a representative $O$. tormota long call (Feng et al. 2009b) from recordings made in 2002 from the same field site that provided the animals in the current experiments (Narins et al. 2004). The O. tormota long calls are 
typically tonal or whistle-like, with a fundamental frequency between 5 and $14 \mathrm{kHz}$ and pronounced harmonics that extend into the ultrasonic range (Narins et al. 2004; Feng et al. 2009b). Males of $O$. tormota discriminate behaviorally between the calls of neighbors and those of strangers (Feng et al. 2009a); thus, the 6-year interlude since the call's recording date ensured that the call would be unfamiliar to the experimental males, avoiding potential confounding of the data due to differential behavioral salience of the stimulus. We performed all subsequent stimulus refinements in Audition 2.0 (Adobe, San Jose, CA). First, we resampled the call from 256 to $500 \mathrm{kHz}$ sampling rate and added a silent period to the beginning and end of the call; these steps were suggested by the playbackequipment manufacturer to avoid aliasing or unintended sound generation during playback. We designated the resulting file the full-spectrum (FS) stimulus. To generate the ultrasound-only (US) stimulus, we high-pass filtered (slope: 1,000 dB per octave) the FS stimulus to remove call components below $20 \mathrm{kHz}$. We then amplified the filtered file by $10 \mathrm{~dB}$. The amplification was performed to equalize the average root mean square (RMS) power of the FS and US stimuli. Without this amplification, it would be difficult to determine whether stimulus-evoked egr-l activation patterns were the result of the frequency composition of the stimuli, or due to differences in stimulus sound pressure level. The final FS and US stimuli were approximately $3 \mathrm{~s}$ long and were looped every $15 \mathrm{~s}$ to form 30-min stimulus blocks. For the no-sound (NS) control, we played back a 30-min Adobe file that did not contain any sound.

\section{Acoustic stimulation}

We captured males of $O$. tormota from natural breeding aggregations in Huangshan Hotsprings, China, between 1830 and 2400 hours from April 18-23, 2008. Males were fully mature, and of average body size (31-34 mm; Fei 1999). We housed them singly or in small groups, in plastic bags or small tanks, and kept them in a quiet room with ambient temperature and light. Our experiments were conducted within 2 days of collection. We placed the focal male in an acoustically transparent plastic bag containing a moistened paper towel. To minimize background acoustic stimulation and achieve baseline levels of $e g r-1$ expression, we kept the bag inside a portable sound attenuation chamber containing an ultrasonic speaker (Ultrasonic Speaker Magnat, Avisoft Bioacoustics, Berlin, Germany; freq. resp.: $1-55 \mathrm{kHz} \pm 7 \mathrm{~dB}$ ) for at least $2 \mathrm{~h}$ before experimental treatment (Burmeister et al. 2008). We positioned the frog approximately $12 \mathrm{~cm}$ from the speaker, a distance calibrated for a peak stimulus sound pressure level of ca. $90 \mathrm{~dB}$ SPL. We then randomly chose the FS, US or NS stimulus and delivered it using Avisoft-SASLab
Pro (Version 4.4; Avisoft Bioacoustics) from a PC laptop to the ultrasonic speaker via a portable US playback interface with an integrated D/A converter (UltraSoundGate Player 116; Avisoft Bioacoustics). After stimulating for $30 \mathrm{~min}$, we kept the frog in silence for an additional $30 \mathrm{~min}$ to reach peak egr-1 expression levels (Burmeister et al. 2008) before decapitation. We exposed 10 frogs to each stimulus for a total sample size of 30 animals. Due to tissue damage during processing, we did not include all brains in the data analysis. All animal care procedures were approved by the UCLA Animal Research Committee (protocol no. 094-086-51).

Tissue preparation and in situ hybridization

After decapitation of a stimulated animal, we quickly surgically exposed the dorsal surface of the brain and submerged the head in freshly prepared $4 \%$ paraformaldehyde (diluted from 16\% formaldehyde ampules; Ted Pella, Redding, CA) for $10 \mathrm{~min}$, and then isolated the brain from the skull and removed the meninges. We flash froze the brain tissue in liquid nitrogen in 2-ml tubes containing Tissue-Tek OCT Compound (Sakura Finetek, Torrance, CA) and stored them on dry ice during transport to the United States. We sectioned the brains in the transverse plane on a cryostat in three series at $16 \mu \mathrm{m}$ thickness, and mounted them onto Superfrost Plus slides (Fisher Scientific, Santa Clara, CA). We reverse transcribed ${ }^{35}$ S-labeled egr-1 mRNA probes from plasmids containing a 411-bp fragment of $R$. pipiens egr- 1 cDNA (GenBank accession no. AY682850.1), see below for probe validation. We processed all the slides in a single in situ hybridization to avoid variation between procedures. Slides hybridized with sense probes showed no binding above background.

The in situ hybridization procedure followed the protocol described in Burmeister et al. (2008). Briefly, we fixed the brain sections in freshly prepared $4 \%$ paraformaldehyde for $10 \mathrm{~s}$ and then rinsed them in phosphatebuffered saline followed by $0.1 \mathrm{M}$ triethanolamine (TEA; Sigma, Saint Louis, MO). We neutralized tissue charge in a solution of $0.25 \%$ acetic anhydride (Sigma) in 0.1 M TEA for $9 \mathrm{~min}$. We then washed the slides twice in saline sodium citrate buffer (SSC; Ambion, Austin, TX) and dehydrated them by progressing through single rinses in $50,70,95 \%$ ethanol, and two rinses in $100 \%$ ethanol. After the slides were fully air-dried, we rehydrated the tissues with radiolabeled riboprobe diluted to $3 \times 10^{6} \mathrm{cpm} / \mathrm{ml}$ in $1 \times$ hybridization buffer (Sigma) and $0.1 \mathrm{M}$ dithiothreitol (DTT; Sigma). After hybridization, we coverslipped the slides, placed them horizontally in racks, and incubated them overnight at $65^{\circ} \mathrm{C}$ in a mineral oil bath. All procedures to this point were RNase free. The following day we removed the oil and coverslips by rinsing the slides twice 
in chloroform and twice in SSC with $1 \mu \mathrm{l} / \mathrm{ml}$ DTT. We eliminated non-specific probe binding by washing the tissues in a $65^{\circ} \mathrm{C}$ solution of $50 \%$ formamide (Sigma), $2 \times$ SSC and $1 \mu \mathrm{l} / \mathrm{ml}$ DTT for $1.25 \mathrm{~h}$ followed by two washes in $0.1 \times \mathrm{SSC}$ for $30 \mathrm{~min}$, both at $65^{\circ} \mathrm{C}$. After a final 5-min rinse in $0.1 \times \mathrm{SSC}$ with $1 \mu \mathrm{l} / \mathrm{ml} \mathrm{DTT}$ at room temperature, we dehydrated the slides using the same ethanol progression described above, and then allowed them to thoroughly air dry.

To visualize the bound riboprobe, we processed the slides for autoradiography. We immersed them in Kodak NTB emulsion (Carestream Health, Rochester, NY) in a darkroom, dried them overnight, placed them in lightproof boxes and stored them for 15 days at $4^{\circ} \mathrm{C}$. We then developed the slides with Kodak fixer and developer. We stained the tissue with thionin to visualize the cell bodies and to facilitate the determination of hindbrain and midbrain nuclear boundaries.

Generation of the egr-1 probe

To generate an $R$. pipiens egr-1 probe, we first used degenerate PCR to amplify a fragment of $R$. pipiens egr- 1 following previously described methods (Hoke et al. 2004). Briefly, we isolated total RNA from brain homogenates and synthesized cDNA using a poly-dT primer and Superscript II reverse transcriptase (Invitrogen, Carlsbad, CA). We designed the following degenerate primers using Codehop (Rose et al. 1998): forward, 5-CCT TCC AGG TGC CCA TGA THC CNG A-3; reverse, 5-GGT CGG ACC GGG AGA ART TNC KCA T-3. To amplify the 411-bp fragment, we used the following cycling parameters on a capillary tube Rapidcycler (Idaho Technologies, Idaho Falls, ID) (note that the rapid heat transfer in a capillary tube thermal cycler does not require hold times for denaturing or annealing steps): an initial 2-min step at $94^{\circ} \mathrm{C}$ followed by 40 cycles as follows: denaturing for $0 \mathrm{~s}$ at $94^{\circ} \mathrm{C}$, annealing for $0 \mathrm{~s}$ at $55,54,53,52,51^{\circ} \mathrm{C}$ (three cycles at each temperature), or $50^{\circ} \mathrm{C}$ ( 25 cycles), and extension for $30 \mathrm{~s}$ at $72^{\circ} \mathrm{C}$, concluding with a final extension for $3 \mathrm{~min}$ at $72^{\circ} \mathrm{C}$. We purified the amplified band and generated plasmids by transforming pCR-II Topo cells (Invitrogen). We verified that the fragment represented $R$. pipiens egr-1 by comparing sequence identity to $e g r-1$ of other vertebrates using BLAST. We found that the $R$. pipiens egr-l sequence shared over $85 \%$ sequence identity with other frogs (e.g., Physalaemus pustulosus and Xenopus tropicalis) and $80 \%$ with birds (e.g., Columba livia).

Because $e g r-1$ is a transcription factor, it is highly conserved among vertebrates (Burmeister and Fernald 2005; Long and Salbaum 1998), and cross-species probe binding has been successful across orders (Jarvis and Mello 2000; Jarvis et al. 2000). Thus, because R. pipiens and $O$. tormota are confamilial, we expected that our $R$. pipiens probe would have a high probability of hybridizing with $O$. tormota egr-1. To test the validity of using an R. pipiens probe with $O$. tormota, we compared the hybridization of $R$. pipiens sense and antisense probes to $R$. pipiens and $O$. tormota brain tissue under high stringency conditions $\left(65^{\circ} \mathrm{C}\right.$ hybridization and washes, see above). After 3 days of exposure to autoradiographic film, we observed that the antisense probe produced clear binding in O. tormota and $R$. pipiens brains whereas the sense probe did not (Fig. 1).

\section{Quantification of egr-1 expression}

We determined relative expression of $e g r-l$ by calculating silver grain density in the SON, and the Ptor and Ltor of the TS (Fig. 2). We were not able to measure expression in the magnocellular nucleus, the third primary subdivision of the TS (Potter 1965) due to the sparseness of cell density therein, which made it difficult to locate unambiguously. We focused our analysis in the brainstem of $O$. tormota since previous multi- and single-unit electrophysiological
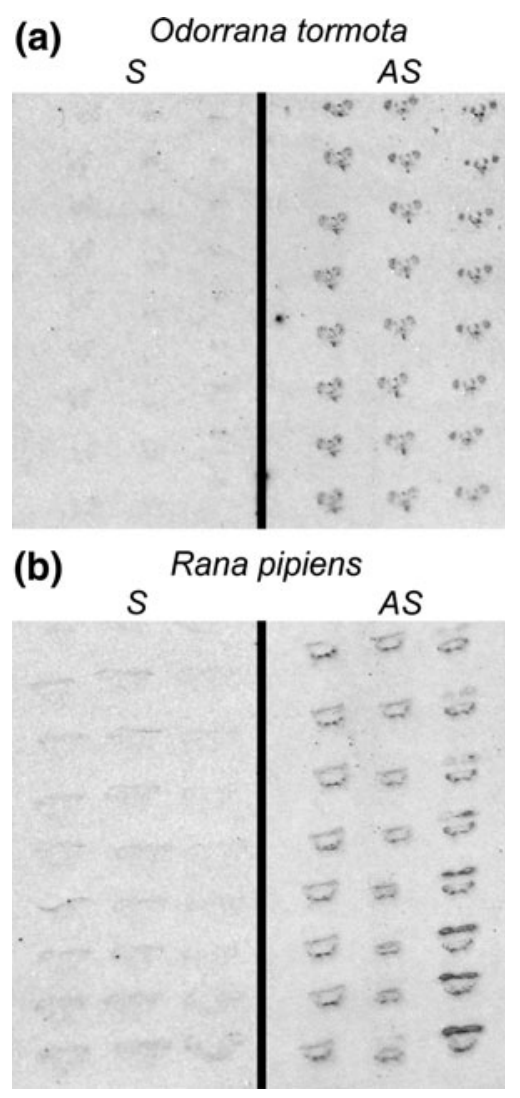

Fig. 1 Image of autoradiographic film showing the results of hybridizing sections of a $O$. tormota and $\mathbf{b} R$. pipiens brain tissue with the sense ( $\mathrm{S}$; left side of figure) and antisense (AS; right side of figure) R. pipiens egr-1 probe. Sections hybridized with the AS probe are darkened, indicating probe binding. Sections hybridized with the $\mathrm{S}$ probe are unlabeled, indicating that the probe did not bind 
Fig. 2 Photomicrographs indicating the approximate location and size of sampling sites in the a (I) laminar nucleus, (II) center of the principal nucleus and (III) dorsal principal nucleus and b (IV) the superior olivary nucleus. Scale bar $0.1 \mathrm{~mm}$. OT optic tectum, $T V$ tectal ventricle, $D T$ dorsal tegmentum, $R$ medullary reticular formation, $V I V$ fourth ventricle

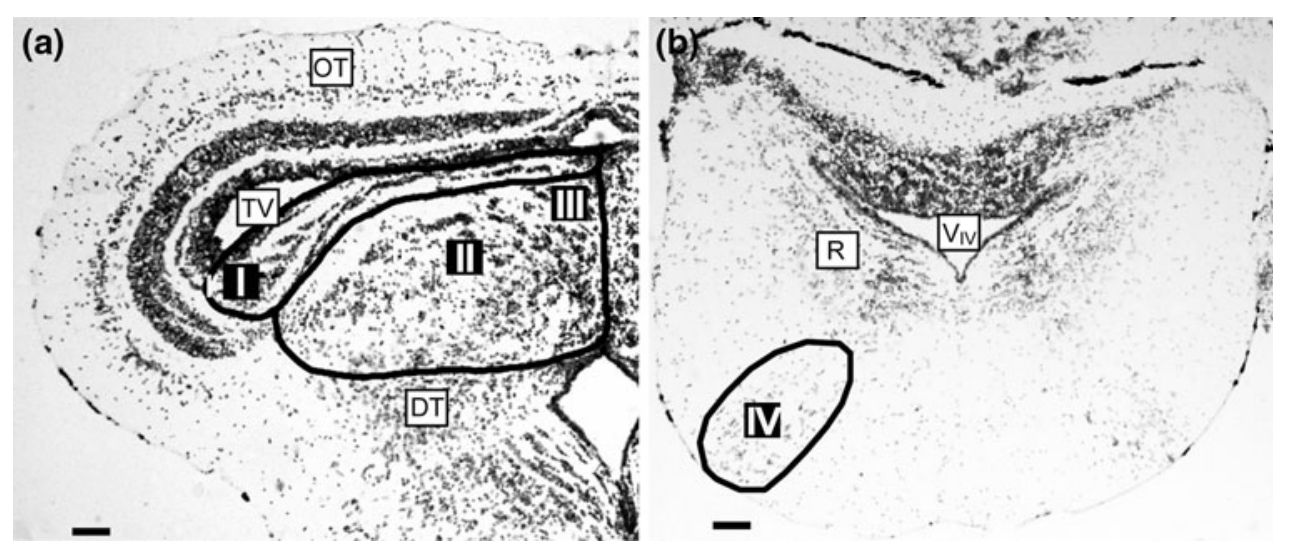

recordings in this species indicated that this region was responsive to ultrasonic stimuli (Feng et al. 2006). We did not analyze egr-l expression in the dorsal medullary nucleus, as sound does not seem to modulate expression of egr-1 in this neural structure in túngara frogs (Chakraborty et al. 2010). We performed all analysis blind to stimulus condition. We identified the nuclei using standard histological landmarks. The Ptor is thought to be tonotopically organized in some frogs (e.g., Rana temporaria, Hermes et al. 1981; Alytes obstetricians, Mohneke 1983; R. pipiens, Feng and Lin 1991), with an "onion-skin" arrangement of isofrequency laminae around a central core (Feng 1983; Mohneke 1983); therefore, we measured egr-l expression levels in a dorsal region (dPtor) and a central region (cPtor) of the nucleus (Fig. 2a) to potentially sample from two different isofrequency contours. We selected the dorsal sampling region to be near the boundary between the Ptor and the laminar cell layers that comprise the Ltor, and the central sampling region to be near the middle of the nucleus. Nuclear boundaries were determined by common landmarks used in the laboratory and related literature, and by referring to a standard atlas of the frog brain (Kemali and Braitenberg 1969). We placed the sampling window for the Ltor in a region where the cells clearly form the parallel layers characteristic to the nucleus (Fig. 2a). For each region, we calculated an individual's mean silver grain density from digital images taken with an $80 \times$ or $100 \times$ objective. The sizes of the sampling windows from these objectives were $102 \times 140 \mu \mathrm{m}$ and $100 \times 120 \mu \mathrm{m}$, respectively. The different sampling windows resulted from our use of two different imaging systems. For each brain region, we used a single imaging system and sampling window. Because our metric for egr-1 expression (see below) was independent of window size, data from different brain regions are comparable. We calculated means from images of three to four sections from the same hemisphere separated by at least $32 \mu \mathrm{m}$. We chose the sampled hemisphere randomly for each individual and brain region. We did not collect data from brains in which nuclei were indistinct due to tissue damage during processing, or from sections that were torn or folded.

We calculated silver grain density above background following procedures described in Burmeister et al. (2005). For each section, we took three images: a green-filtered image that increased the contrast of the thionin-stained cell bodies ('cells image'), a blue-filtered image of only the grains in the same field of view ('grains image') and a bluefiltered image of the slide adjacent to the tissue to measure local background silver grain density ("background image'). Exposure, brightness and contrast settings were the same for both blue-filtered images from a given section. We performed the quantification with ImageJ (National Institutes of Health, Bethesda, MA). First, we converted the grains and background images to binary and counted the silver grains in each image using the "Analyze Particles" function with a minimum particle size of 1 pixel. We then subtracted the number of background silver grains from the number in the region of interest to measure the number of silver grains above background per image. We calculated the area covered by cell bodies from the "cells image". Our final measurement of $e g r-1$ expression was calculated as the ratio of silver grains above background to pixels covered by cells.

Since our data were not normally distributed, we used the Kruskal-Wallis test to probe for an effect of stimulus type on egr-1 expression levels in the four sampling regions. We then did pair-wise comparisons of the stimuli effects using the Mann-Whitney $U$ test.

\section{Results}

Mating calls induced expression of egr- 1 in the SON and cPtor and dPtor of the TS, but not in the Ltor (Table 1; Fig. 4). In the SON, presentations of the full-spectrum (FS) and high-pass filtered, ultrasound-only (US) calls evoked similar levels of egr-1 expression, which were significantly higher than expression levels seen in response to the NS 
Table 1 Sample sizes (number of brains) for the three stimuli in each sampled brain region, and the results of non-parametric analysis of variance and pair-wise statistical tests

\begin{tabular}{|c|c|c|c|c|c|c|c|}
\hline \multirow{3}{*}{ Brain region } & \multicolumn{3}{|c|}{ Sample size } & \multicolumn{4}{|l|}{$P$ values } \\
\hline & \multirow[t]{2}{*}{ FS } & \multirow[t]{2}{*}{ US } & \multirow[t]{2}{*}{$\mathrm{NS}$} & \multirow{2}{*}{$\begin{array}{l}\text { Kruskal-Wallis } \\
\text { analysis of variance }\end{array}$} & \multicolumn{3}{|c|}{ Mann-Whitney } \\
\hline & & & & & $\mathrm{FS} \times \mathrm{US}$ & $\mathrm{FS} \times \mathrm{NS}$ & $\mathrm{US} \times \mathrm{NS}$ \\
\hline Superior olive & 8 & 8 & 9 & 0.048 & 0.645 & 0.036 & 0.046 \\
\hline Laminar & 7 & 10 & 8 & 0.402 & 0.813 & 0.281 & 0.274 \\
\hline Dorsal principal & 6 & 8 & 8 & 0.063 & 0.197 & 0.156 & 0.036 \\
\hline Center principal & 5 & 8 & 8 & 0.048 & 0.057 & 0.884 & 0.027 \\
\hline
\end{tabular}

Italics indicate statistical significance at $P<0.05$ level

FS full spectrum, US ultrasound only, NS no sound
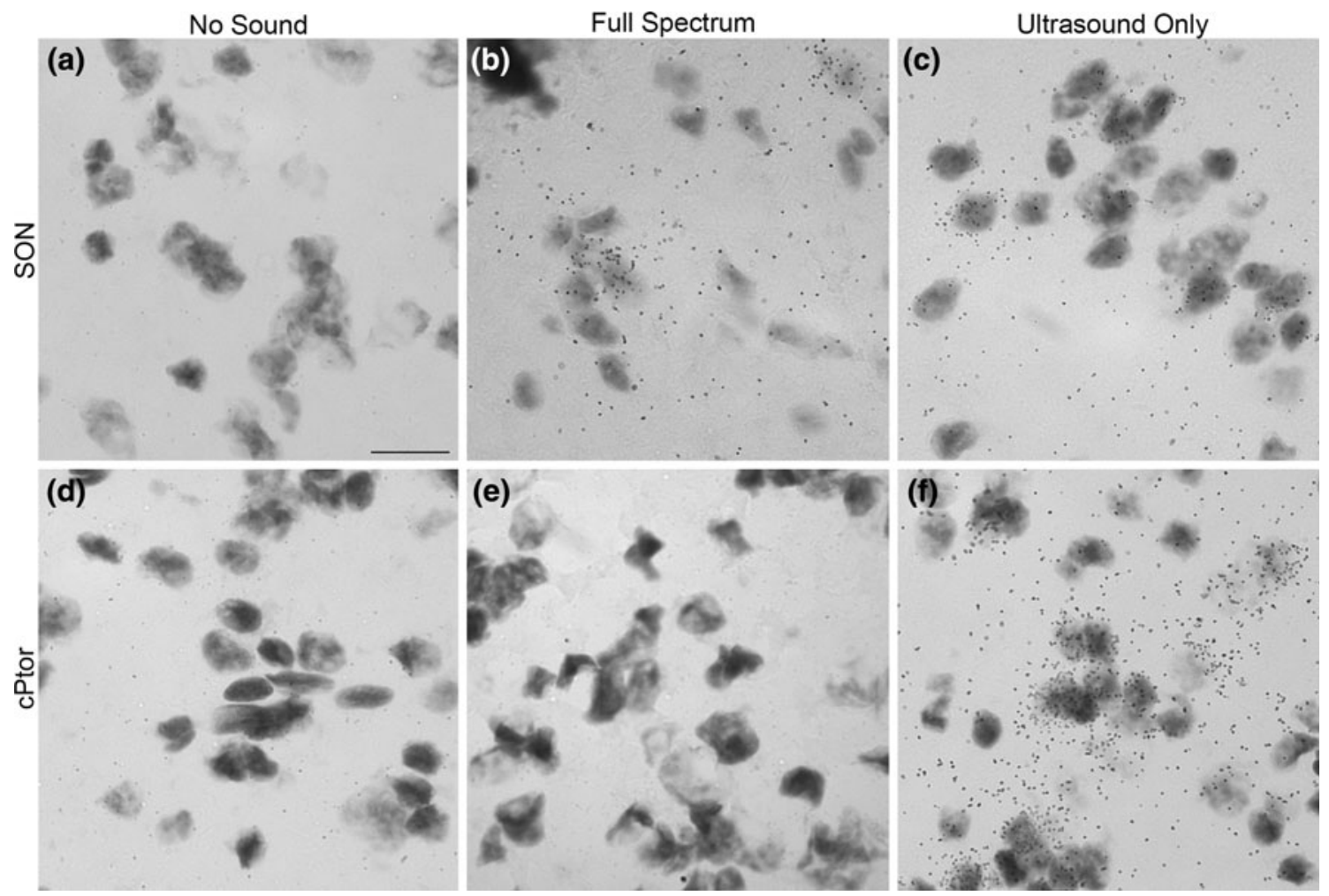

Fig. 3 Photomicrographs showing egr-1 mRNA levels in response to the no-sound control, and full-spectrum and ultrasound-only stimuli in a-c the superior olivary nucleus (SON) and $\mathbf{c}-\mathbf{f}$ the central sampling site in the principal nucleus (cPtor). Scale bar $20 \mu \mathrm{m}$

control (Table 1; Figs. 3, 4a). Typically, egr-1 was expressed at moderate levels in a small number of cells within the nucleus (Fig. 3). In contrast, in the sampled regions of the Ptor, the US stimulus appeared to evoke more egr-1 expression than the FS call, although the contrast between these stimuli did not quite reach statistical significance (Table 1; Figs. 3, 4c). The weakness of this effect may be due to constraints on our sample size imposed when working with this species. In the cPtor and dPtor, egr-l was expressed in a larger proportion of the cells compared to the SON (Fig. 3). Remarkably, the FS call elicited almost no egr-1 expression in the sampled regions of the Ptor (Fig. 3), in contrast to the túngara frog where natural, full-spectrum calls elicit robust egr-1 expression in the Ptor (Chakraborty et al. 2010). Interestingly, the trend of higher egr-1 expression in response to the US stimulus was particularly evident for the cPtor sampling site (Table 1; Figs. 3, 4d). In this region, US evoked higher egr-1 expression compared to the NS control, while the $e g r-l$ expression level following FS stimulation was no different from that of the control (Fig. 4c). For the Ltor of the TS, egr-1 expression following FS or 
Fig. 4 Fold change in mean egr-1 expression $(+\mathrm{SE})$ relative to the no sound (NS) stimulus in a the superior olivary nucleus (SON), b the laminar nucleus (Ltor), $\mathbf{c}$ the dorsal principal nucleus (dPtor) and $\mathbf{d}$ the center of the principal nucleus (cPtor). Numbers indicate pair-wise significance. Significant alpha values $(P<0.05)$ are italicized. US ultrasound-only stimulus, FS full-spectrum stimulus

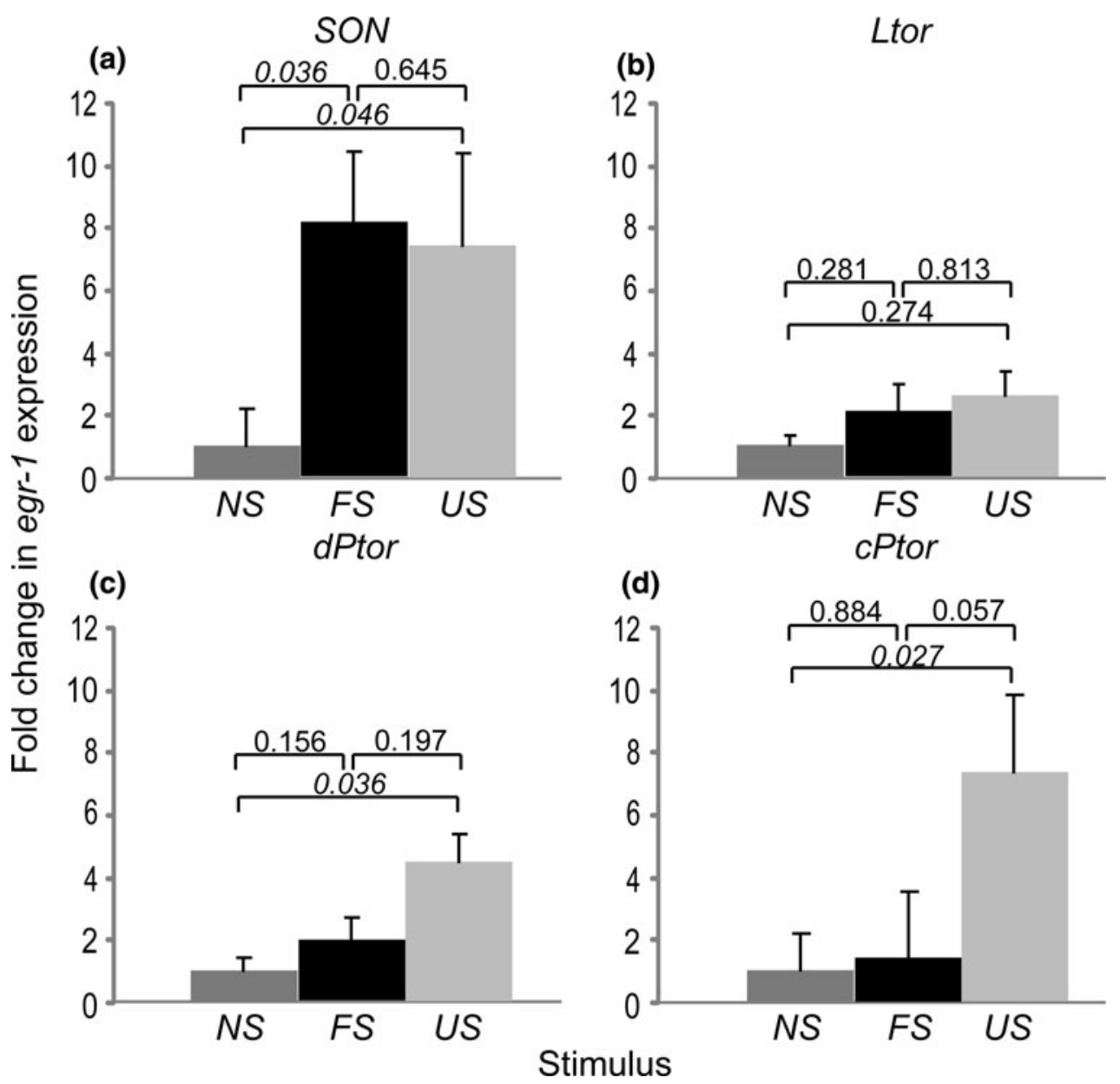

US stimulation was no different from that of the control, and FS and US induced similar levels of egr- 1 expression (Table 1; Fig. 4b).

\section{Discussion}

Previous studies have characterized mating-call induced expression of egr-1 in the brainstem of the túngara frog (e.g., Hoke et al. 2004; Chakraborty et al. 2010), but ours is the first to test the effect of spectrally manipulated calls on egr-1 expression. As in the túngara frog, we found that mating calls increased egr-1 expression in both the SON and Ptor (Chakraborty et al. 2010), although we did not detect an effect of mating calls on egr-1 expression in Ltor. In the SON, we found that FS and US calls induced comparable egr-1 expression levels. However, in the Ptor, we found that the US call evoked the highest level of egr-1 expression, although the difference did not quite reach statistical significance. The trends in the data indicate that the Ptor may be quite sensitive to ultrasound.

Based on cytoarchitecture, the TS in O. tormota and $R$. pipiens closely resembles each other. We found no cytoarchitectural evidence for an additional nucleus within the $O$. tormota brain. This suggests that a large-scale reorganization of the nuclei within the $O$. tormota central auditory system is not necessary for these frogs to accommodate ultrasound sensitivity. The overt egr-1 expression in the SON in response to the US stimulus indicates that ultrasounds are relayed to the auditory midbrain via this intermediate nucleus, as is true of frequencies within the typical anuran hearing range.

In the midbrain, egr-1 expression patterns in response to the FS and US stimuli were more complex than in the hindbrain. Egr-1 levels in the sampled areas of the Ptor showed a trend toward higher expression in response to the US versus the FS stimulus. This trend was stronger centrally than dorsomedially, suggesting that the frogs have a greater proportion of high-frequency-sensitive neurons toward the middle of the nucleus (Figs. 3, 4d).

Although the trends toward difference in egr-1 expression levels for US and FS stimulation did not reach statistical significance in the Ptor (Table 1; Fig. 4), this may be due to limited sample size; the sample sizes of the groups were necessarily small due to constraints on access to the animals, reducing the power of the analysis and 
limiting our ability to include a fourth experimental group in which the frogs were presented with the audible components of the call only. We chose to use a full-spectrum call as one of our stimuli in order to explore the brain's response to the unaltered conspecific signal but, as a result, the two experimental groups in our study did not have complete spectral contrast. Both experimental stimuli contained some ultrasonic components, although they were considerably amplified in the US stimulus to ensure that the RMS sound pressure of the two stimuli was equal (see "Methods"). Future experiments could further probe the functional networks of the frogs' auditory system by employing sound stimuli with greater spectral contrast, e.g., by low-pass and high-pass filtering a conspecific call.

The apparent ability of our sampled areas of the Ptor in O. tormota to encode high frequencies suggests that highfrequency call components play a key role in the species' communication system. The behavioral importance of this spectral subset of the frogs' vocalizations was substantiated by the males' strong response to the broadcast of high-pass filtered conspecific calls (Feng et al. 2006). We hypothesize that the frogs' attention to high-frequency call components may have evolved to facilitate sound localization. Interaural intensity cues are only available if an animal hears frequencies with wavelengths short enough to be shadowed by its head. O. tormota has an interaural distance of $<1 \mathrm{~cm}$, yet has remarkable sound localization ability, with a localization error of $<1^{\circ}$ (Shen et al. 2008). This hyperacuity is extraordinary among amphibians (Christensen-Dalsgaard 2005), and suggests that this species uses its unusual high-frequency sensitivity to facilitate localization (Shen et al. 2008). The selective benefits of accurate localization provide a powerful evolutionary rationale for the specialization of regions of the $O$. tormota Ptor for high-frequency encoding. Similarly, dedicated brain regions are seen across species that are specialized for processing particular types of auditory information (Suga 1989), such as the barn owl's (Tyto alba) nucleus mesencephalicus lateralis dorsalis (Knudsen and Konishi 1978) and the dorsoposterior division of the mustache bat (Pteronotus parnelli) inferior colliculus (Pollak et al. 1986). In both of these species, as in $O$. tormota, the basic template of the taxon's central auditory system has been retained; however, functional subdivisions are specialized for processing particular types of auditory information critical to the organism's survival.

In conclusion, $O$. tormota has evolved the extraordinary ability to detect high-frequency sounds, a sensory domain previously thought to be exclusively mammalian among the vertebrates. This species therefore provides an interesting comparative system for exploring the integration of high-frequency sensitivity into the peripheral and central vertebrate auditory systems. Our analysis of egr-1 expression indicates that there are high-frequency-sensitive neurons within the Ptor of the TS, suggesting that this frogs' auditory midbrain is specialized for high-frequency encoding.

Acknowledgments We are grateful to Christina Lebonville for her assistance with the in situ hybridization and data analysis. Dr. Lisa Mangiamele provided helpful advice and insight during the laboratory work. Drs. Keith Sockman and Niranjala Tillakaratne generously allowed the use of their microscopes and cameras for data collection. Marichris Nario assisted with data collection and analysis. Financial support was provided by grants from the National Institutes of Health, National Institute on Deafness and Other Communication Disorders (no. DC-00222) and the Paul S. Veneklasen Research Foundation to PMN, a National Science Foundation Doctoral Dissertation Improvement Grant (no. 0806207) to VSA, a National Science Foundation Grant (no. IOB0445682) to SSB, and a grant from the National Natural Sciences Foundation of China (no. 30730029) to JXS. We thank Dr. Stephen Arch for helpful comments on the manuscript.

Open Access This article is distributed under the terms of the Creative Commons Attribution Noncommercial License which permits any noncommercial use, distribution, and reproduction in any medium, provided the original author(s) and source are credited.

\section{References}

Arch VS (2010) The neuroethology of ultrasonic communication in anuran amphibians. Ph.D. Dissertation. University of California

Burmeister SS, Fernald RD (2005) Evolutionary conservation of the egr-1 immediate early gene response in a teleost. J Comp Neurol 481:220-232

Burmeister SS, Jarvis ED, Fernald RD (2005) Rapid behavioral and genomic responses to social opportunity. PLoS Biol 3(11):e363

Burmeister SS, Mangiamele LA, Lebonville CL (2008) Acoustic modulation of immediate early gene expression in the auditory midbrain of female túngara frogs. Brain Res 1190:105-114

Chakraborty M, Mangiamele LA, Burmeister SS (2010) Neural activity patterns in response to interspecific and intraspecific variation in mating calls in the túngara frog. PLoS ONE 5(9):e12898

Christensen-Dalsgaard J (2005) Directional hearing in nonmammalian tetrapods. In: Popper AN, Fay RR (eds) Sound source localization. Springer, New York, pp 67-123

Clayton DF (2000) The genomic action potential. Neurobiol Learn Mem 74:185-216

Fei L (1999) Atlas of amphibians of China. Henan Science and Technology Press, Zhengzhou

Feng AS (1983) Morphology of neurons in the torus semicircularis of the northern leopard frog, Rana pipiens pipiens. J Morphol 175:253-269

Feng AS, Lin W (1991) Differential innervation patterns of three divisions of frog auditory midbrain (torus semicircularis). J Comp Neurol 306:613-630

Feng AS, Narins PM, Xu C-H, Lin W-Y, Yu Z-L, Qiu Q, Xu Z-M, Shen J-X (2006) Ultrasonic communication in frogs. Nature 440:333-336

Feng AS, Arch VS, Yu Z-L, Yu X-J, Xu Z-M, Shen J-X (2009a) Neighbor-stranger discrimination in concave-eared torrent frogs, Odorrana tormota. Ethology 115:851-856 
Feng AS, Riede T, Arch VS, Yu Z-L, Xu Z-M, Shen J-X (2009b) Diversity of the vocal signals of concave-eared torrent frogs (Odorrana tormota): evidence for individual signatures. Ethology 115:1015-1028

Fettiplace R, Fuchs PA (1999) Mechanisms of hair cell tuning. Ann Rev Physiol 61:809-834

Hermes DJ, Aertsen AM, Johannesma PI, Eggermont JJ (1981) Spectro-temporal characteristics of single units in the auditory midbrain of the lightly anaesthetised grass frog (Rana temporaria $\mathrm{L}$ ) investigated with noise stimuli. Hear Res 5:147-178

Hoke KL, Burmeister SS, Fernald RD, Rand SA, Ryan MJ, Wilczynski W (2004) Functional mapping of the auditory midbrain during mate call reception. J Neurosci 24:11264-11272

Jarvis ED (2004) Brains and birdsong. In: Marler P, Slabbekoorn H (eds) Nature's music: the science of birdsong. Elsevier-Academic, New York, pp 239-275

Jarvis ED, Mello CV (2000) Molecular mapping of brain areas involved in parrot vocal communication. J Comp Neurol 419:1-31

Jarvis ED, Ribeiro S, da Silva ML, Ventura D, Vielliard J, Mello CV (2000) Behaviourally driven gene expression reveals song nuclei in hummingbird brain. Nature 406:628-632

Kemali M, Braitenberg V (1969) Atlas of the frog's brain. Berlin, Springer

Knudsen E, Konishi M (1978) A neural map of auditory space in the owl. Science 200:795-797
Long KD, Salbaum JM (1998) Evolutionary conservation of the immediate-early gene ZENK. Mol Biol Evol 15:284-292

Mohneke R (1983) Tonotopic organisation of the auditory midbrain nuclei of the midwife toad (Alytes obstetricans). Hear Res 9:91-102

Narins PM, Feng AS, Lin W-Y, Schnitzler HÜ, Denzinger A, Xu C-H (2004) Old World frog and bird vocalizations contain prominent ultrasonic harmonics. J Acoust Soc Am 115:910-913

Pollak G, Wenstrup J, Fuzessery Z (1986) Auditory processing in the mustache bat's inferior colliculus. Trends Neurosci 9:556-561

Potter HD (1965) Mesencephalic auditory region of the bullfrog. J Neurosci 28:1132-1154

Rose TM, Schultz ER, Henikoff JG, Pietrokovski S, McCallum CM, Henikoff S (1998) Consensus-degenerate hybrid oligonucleotide primers for amplification of distantly related sequences. Nucleic Acids Res 26:1628-1635

Shen J-X, Feng AS, Xu Z-M, Yu Z-L, Arch VS, Yu X-J, Narins PM (2008) Ultrasonic frogs show hyperacute phonotaxis to female courtship calls. Nature 453:914-916

Suga N (1989) Principles of auditory information-processing derived from neuroethology. J Exp Biol 146:277-286

Wilczynski W (1988) Brainstem auditory pathways in anuran amphibians. In: Fritzsch B, Ryan MJ, Wilczynski W, Hetherington TE, Walkowiak $\mathrm{W}$ (eds) The evolution of the amphibian auditory system. Wiley, New York, pp 209-231 